\title{
Simulation of Earthquakes with Cellular Automata
}

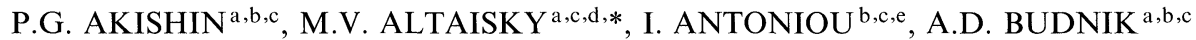 \\ and V.V. IVANOV ${ }^{\mathrm{a}, \mathrm{b}, \mathrm{c}}$ \\ a Laboratory of Computing Techniques and Automation, Joint Institute for Nuclear Research, Dubna, 141980, Russia; \\ ${ }^{\mathrm{b}}$ International Solvay Institutes for Physics and Chemistry, CP-231, ULB, Campus Plaine, Bd. du Triomphe, 1050, Brussels,

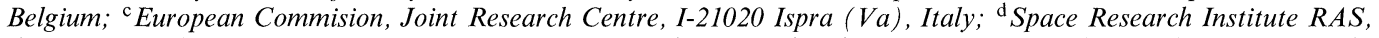 \\ Profsoyuznaya 84/32, Moscow, 117810, Russia; ${ }^{\mathrm{e}}$ Theoretische Natuurkunde, Free University of Brussels, Brussels, Belgium
}

(Received 20 April 1998)

\begin{abstract}
The relation between cellular automata (CA) models of earthquakes and the BurridgeKnopoff (BK) model is studied. It is shown that the CA proposed by $P$. Bak and C. Tang, although they have rather realistic power spectra, do not correspond to the BK model. We present a modification of the $\mathrm{CA}$ which establishes the correspondence with the BK model. An analytical method of studying the evolution of the BK-like $\mathrm{CA}$ is proposed. By this method a functional quadratic in stress release, which can be regarded as an analog of the event energy, is constructed. The distribution of seismic events with respect to this "energy" shows rather realistic behavior, even in two dimensions. Special attention is paid to twodimensional automata; the physical restrictions on compression and shear stiffnesses are imposed.
\end{abstract}

Keywords: Earthquakes, Cellular automata

\section{INTRODUCTION}

The dynamics of earthquake faults is receiving a lot of attention as an interdisciplinary problem, which seems to manifest a great deal of all known types of chaotic behavior. At the same time, the question whether earthquake faults are predictable is still open. Bak and Tang [1] suggested that earthquake faults seem to be physical manifestations of selforganized criticality (SOC) [2]. This observation was mainly based on the Gutenberg-Richter law

$$
\log N=a-b m,
$$

which relates the cumulative number of events $N$ with seismic moment greater than the value $M$, to the seismic magnitude $m=\log M$. Since $M$ is proportional to the energy of the seismic event $\log E=c-d m$, the logarithmic law (1), empirically found from the earthquake statistics [3], can be

\footnotetext{
* Corresponding author. Laboratory of Computing Techniques and Automation, Joint Institute for Nuclear Research, Dubna, 141980, Russia. E-mail: altaisky (a) linserv.jinr.ru.
} 
expressed in terms of earthquake energies: $N\left(E>E_{0}\right) \sim E^{-\tau} ; a, b, c, d$ are empirical constants.

The power distribution of seismic events is rather nontrivial fact: from elementary thermodynamics one can expect that the density of events behaves like $\exp (-E / T)$. The power distribution found in the seismic events has attracted new attention to the SOC systems [2] for which the power-law behavior is common. Such systems are remarkable that they constantly evolve to some minimally stable state, and in this sense are stabilized in an unstable state. The simplest system which shows SOC behavior is the sand pile. Later, the same phenomenon has been observed in other complex systems and was found to go along with so-called flicker-noise, a noise with $1 / f$ power spectrum.

The peculiarity of the earthquakes, if considered as a SOC phenomena, is the coexistence of two aspects of the earthquake problem. On one hand, the earthquakes can be considered as a fracture propagation process in the earth's crust. The earth's crust has fractal geometry [4] and that is why the power law distribution of observed characteristics is strongly expected here [5]. On the other hand, the dynamical modelling of earthquake faults is usually performed only on a small fragment of the crack network, so locally the fault is reduced to slip and stick behavior of two Euclidean half-space domains. The dynamics of this small zoom of the fragmentating crust is introduced by the assumption that one of the half-spaces is divided into blocks connected by springs: this is the Burridge-Knopoff $(B K)$ ansatz. The complex hehavior of the BK system [6] can by no means be attributed to the global self-similarity of crust fragmentation. However, some properties of the BK model can be extrapolated to the behavior of the rupturing media with domain larger than that of simulated flat fault.

The advantage of the distributed computational systems for such extrapolation is apparent: Even for numerical simulation of the dynamics of spring-block systems of a few hundred blocks hours of CPU time of modern computer are required. For 2D or even 3D simulation of the rupture propagation, a system of $N^{D}$ differential equations should be solved. The application of cellular automata $(C A)$ seems very attractive in this sense, since the process is evaluated by CA locally, and hence goes fast.

The remainder of this paper is organized as follows. In Section 2 we review the principles of constructing BK-like CA, show that their symmetry does not pertain that of the BK model, propose a modification of the CA rules which eliminates this discrepancy. In Section 3 we analyze the problem of simulating tectonic processes with CA. It is shown that for more realistic modelling of earthquakes, in order to account for the shear stresses, both static and dynamic variables should be simulated by the CA. An analytical method which enables us to give a new definition of a CA-simulated event magnitude is constructed. Section 4 is devoted to the two-dimensional aspects of the stress redistributing cellular automata (SRCA). We discuss in the Conclusion the results of numerical simulations, present statistical characteristics of the CA-simulated time series and highlight further investigations.

\section{BK-LIKE CELLULAR AUTOMATA: PRINCIPLES OF CONSTRUCTION}

The power-law distribution of events, observed for both the SOC systems [1] and the real earthquakes (the Gutenberg-Richter law), has stimulated interest to the sand-pile-like CA as a model for real earthquakes. Here we review the basic ideas and principles.

\subsection{The Idea of Self-Organized Criticality}

Following the original paper [2] let us consider a one-dimensional array of $N$ particles. Let $z_{n}=$ $h_{n}-h_{n+1}$ represent the height differences of neighboring positions. The SOC dynamics unfolds as follows:

1. Adding a particle to the $n$th site we increase the right difference and decrease the left difference:

$$
z_{n} \rightarrow z_{n}+1, \quad z_{n-1} \rightarrow z_{n-1}-1 .
$$


2. If the height difference exceeds certain critical value, one block tumbles to the left (lower) position:

$$
z_{n} \rightarrow z_{n}-2, \quad z_{n \pm 1} \rightarrow z_{n \pm 1}+1
$$

3. The boundary conditions provide free fall from the edges:

$$
\begin{aligned}
& z_{0}=0, \quad \text { and } \quad z_{N} \rightarrow z_{N}-1 \text {, for } z_{N}>z_{\mathrm{c}} \\
& z_{N-1} \rightarrow z_{N-1}+1 \text {. }
\end{aligned}
$$

The redistribution law (2) is usually referred to as a sand-pile cellular automaton.

\subsection{Burridge-Knopoff Model and Stress Redistribution}

The BK dynamical model of earthquake faults is a chain of $N$ blocks of mass $m_{i}, i=1, \ldots, N$ resting on a rough surface and connected by harmonic springs of stiffness $k_{\mathrm{c}}$ to each other; each block is attached by a leaf spring of stiffness $k_{\mathrm{p}}$ to the moving upper line, see Fig. 1.

Initially (at $t=0$ ) the system is at rest and the elastic energy accumulated in the "horizontal" springs is only due to randomly generated small initial displacements of the blocks from their neutral positions. The moving upper line, which simulates the movement of the external driving plate (the tectonic stress), exerts the force $f_{\mathrm{n}}=$ $-k_{\mathrm{p}}\left(x_{\mathrm{n}}-v t\right)$ on each block. The nonlinear friction is defined in such a way that it holds each block at rest until the sum of all forces applied to this block exceeds certain critical value $f_{\text {th }}$ - then the block

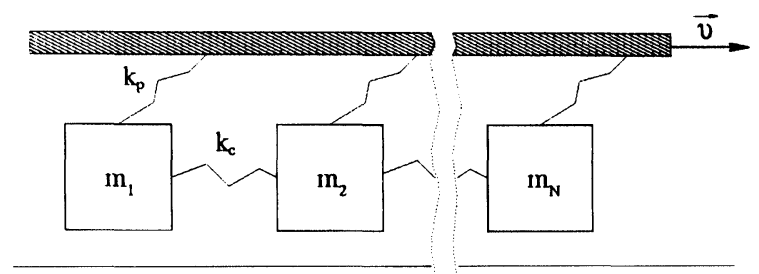

FIGURE 1 The geometry of the BK model. The system is composed of $N$ identical blocks of mass $m_{i}, k_{\mathrm{c}}$ is the stiffness of the "horizontal" springs, $k_{\mathrm{p}}$ is the stiffness of the pulling springs, $v$ is the constant velocity of the pulling line. makes a slip to a new position with less energy. Depending on the particular stress distribution amongst the chain springs in the current event, a one block slip, a number of blocks slip or a global slip of all system may happen. Due to the particular form of nonlinear friction force [6,7] the new block position after a slip is of much lower potential energy than in its previous position. The duration of an event (a quake) in the BK model is understood as a time-period during which the velocity of at least one block exceeds $v$.

A simple CA analog of the BK spring-block model was proposed by Bak and Tang in [1]. Their model consists of an array of particles $(0 \leq i \leq N)$ subjected to the reaction force from their neighbors and constantly applied driving force - the "tectonic force". Let $f_{\text {th }}$ be the threshold value after which the stress is relaxed. Then, for such $i$ that $f_{i}>f_{\text {th }}$, where $i$ is the block number, the stress redistribution law

$$
f_{i} \rightarrow f_{i}-2, \quad f_{i \pm 1} \rightarrow f_{i \pm 1}+1
$$

is applied. The generalization to two dimensions is straightforward [1].

Evidently this force redistribution law is too simple and by no means corresponds to the BK model, where the force (stress) dynamics is governed by a second-order differential equation.

\subsection{The Nakanishi CA}

A more elaborated construction of a BK-like CA has been proposed by Nakanishi [8]. Starting from the equation of motion of the BK system [6]

$$
\begin{aligned}
m \ddot{x}_{i}= & k_{\mathrm{c}}\left(x_{i+1}+x_{i-1}-2 x_{i}\right) \\
& +k_{\mathrm{p}}\left(v t-x_{i}\right)+f_{\text {diss }}\left(\dot{x}_{i}\right),
\end{aligned}
$$

which determines the motion of the $i$ th block, it is easy to express the stress (i.e. the sum of forces applied to each block of the system) before and after the event. Let $x_{i}$ be the displacement of $i$ th block from its neutral position before the slip, $x_{i}^{\prime}$ be its position after the slip, then the total forces 
exerted by this block are

$$
\begin{aligned}
& f_{i}=k_{c}\left(x_{i+1}+x_{i-1}-2 x_{i}\right)+k_{\mathrm{p}}\left(v t-x_{i}\right), \\
& f_{i}^{\prime}=k_{c}\left(x_{i+1}+x_{i-1}-2 x_{i}^{\prime}\right)+k_{\mathrm{p}}\left(v t^{\prime}-x_{i}^{\prime}\right),
\end{aligned}
$$

before and after the slip, respectively. The stress released in event is

$$
\delta f_{i}=f_{i}-f_{i}^{\prime}=\left(2 k_{\mathrm{c}}+k_{\mathrm{p}}\right)\left(x_{i}^{\prime}-x_{i}\right) .
$$

The duration of the event $\left(\Delta t=t^{\prime}-t\right)$, is ignored at this stage but is applied at the final stage of CA evolution as described below.

If the force $f_{i}$ exceeds the threshold value $f_{\text {th }}$ and the $i$ th block makes a slip, the release of stress (5) is assumed to be equally shared between its neighbors:

$$
f_{i \pm 1} \rightarrow f_{i \pm 1}+\frac{k_{\mathrm{c}}}{2 k_{\mathrm{c}}+k_{\mathrm{p}}} \delta f_{i}
$$

The stress of the $k_{\mathrm{p}}$-springs is not shared. The redistribution law (6) does not follow from the dynamics of the BK system (3) - the new position $x_{i}^{\prime}$ is unknown - but is believed to be physically acceptable.

In Nakanishi's paper, the new after-slip value of stress $f_{i}^{\prime}$, is supposed to obey a deterministic evolution law

$$
f_{i}^{\prime}=\phi\left(f_{i}-f_{\mathrm{th}}\right)
$$

To complete the CA cycle "the tectonic stress" is added to all blocks:

$$
f_{i}^{\prime} \rightarrow f_{i}^{\prime}+k_{\mathrm{p}} v \Delta t
$$

In this way the duration of a single event $\Delta t$ becomes a parameter of the Nakanishi CA.

Referring the reader to the original paper [8] for general discussion concerning the exact form of the relaxation function $\phi(x)$ and its parameters, we just present its specific form used in our numerical simulations:

$$
\phi(x)=\frac{(2-\delta f)^{2} / \alpha}{x+(2-\delta f) / \alpha}-1
$$

The plot of the function (9) for specific values of the stress-gap parameter $\delta f=0.01$ and a number of different values of $\alpha$ is presented in Fig. 2 .

The one-dimensional cellular automaton with the relaxation function (9) shows the event distribution behavior which may be described as a logarithmic law (1) with $b=0.84$. This distribution is shown in Fig. 3. The magnitude was defined as a logarithm of the stress relaxation in the event

$$
m=\log M, \quad M=\sum_{i}\left(f_{i}^{\prime}-f_{i}\right)
$$

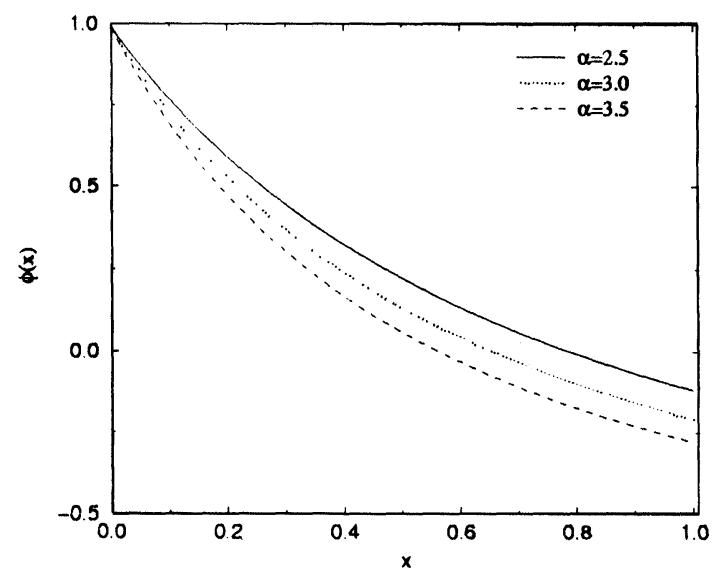

FIGURE 2 The stress relaxation function $\phi: f^{\prime}=\phi\left(f-f_{\mathrm{th}}\right)$, taken from Nakanishi [8]; plotted here for $\alpha=2.5,3,3.5$.

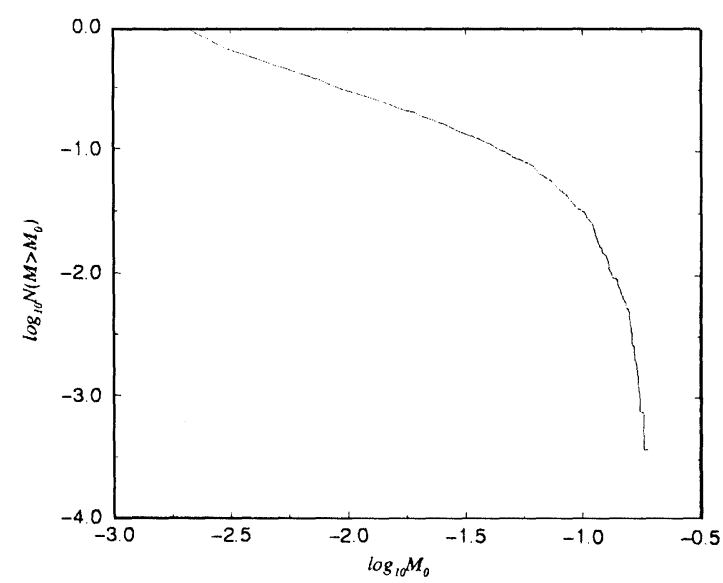

FIGURE 3 Cumulative event distribution obtained with Nakanishi's cellular automaton for 35 blocks, $\alpha=3, \delta f=0.01$, $\Delta t=0.85$. 
This is in agreement with that for the BK model [7], where $M_{\mathrm{BK}}=\sum_{i}\left(x_{i}^{\prime}-x_{i}\right)$, if $f^{\prime}-f \approx k_{\mathrm{p}}\left(x^{\prime}-x\right)$.

\section{EARTHQUAKE EVOLUTION AND CA DYNAMICS}

Considering the dynamics of the cellular automata $[1,8]$, one has to admit at least a few aspects in which these CA are different from real tectonic processes and even from the BK model. First, in contrast to the BK model, the CA dynamics is the dynamics of stress redistribution: no dynamical variables like velocities are included. Second, the definition of the magnitude as a difference of stresses before and after the event is not strictly adopted for these CA. Third, one of the basic principles of the construction of cellular automata for physical applications, is the preservation of symmetry of the original physical system as well as possible. Both, Bak and Tang [1] and Nakanishi [8] CA do not meet these requirements.

We introduce a new CA which is an extension of the Bak and Tang and Nakanishi CA and takes into account these three requirements.

\subsection{Investigation of the CA Dynamics}

If the magnitude of a real seismic event is more or less well defined quantity - the decimal logarithm of displacement measured at certain gauge distance from the epicenter - then, on contrary, for the Bak and Tang CA [1], or even for more advanced Nakanishi's [8] automaton, it is not clear completely, why the logarithm of the sum of tensions redistributed by the cellular automaton should be regarded as a magnitude. (This problem does not arise, say, for the BK model, where displacements and forces are related by a given system of differential equations.)

So, regardless of the fact that SOC systems give a power-law distribution of events, we have to admit that neither the Bak and Tang CA [1] nor the Nakanishi's CA [8] accurately account for the dynamical part of the problem (displacements and velocities), but deal only with static parts (the tensions). Therefore, just a bare analogy between the BK model and the Nakanishi CA does not form a reliable basis for the identification of the magnitude of seismic events with that of tensions redistributed by the cellular automaton.

The only quantities we have at our disposal dealing with stress redistributing $\mathrm{CA}$ are stress release $\sum_{k}\left(f_{k}^{\prime}-f_{k}\right)$ and stress accumulation $\sum_{k} f_{k}$. We do not have any kinetic characteristics, and the only thing we can do is to use the function $f^{\prime}=\phi\left(f-f_{\text {th }}\right)$. Doing so, we construct an analog of the kinetic energy

$$
E(t)=\frac{1}{N} \sum_{k=1}^{N}\left[\phi\left(f_{k}(t)-f_{\mathrm{th}}\right)-f_{k}(t)\right],
$$

The averaged accumulated stress $F(t)=1$ / $N \sum_{k=1}^{N} f_{k}(t)$ at the r.h.s. of Eq. (11), can he regarded as a control parameter, which shows the vicinity of the current CA state to the critical state. Hereafter we shall call $E(t)$ and $F(t)$ the kinetic and potential function, respectively.

In the figures below the time behavior of $E(t)$ and $F(t)$ is presented. In our simulations we used onedimensional CA of $(N=) 35$ cells with relaxation function (9). The initial configuration was given by homogeneous random distribution of $f_{i}: 0 \leq f_{i} \leq f_{\text {th }}$, $i=1, \ldots, N$. As it can he seen from Fig. 4(a), during

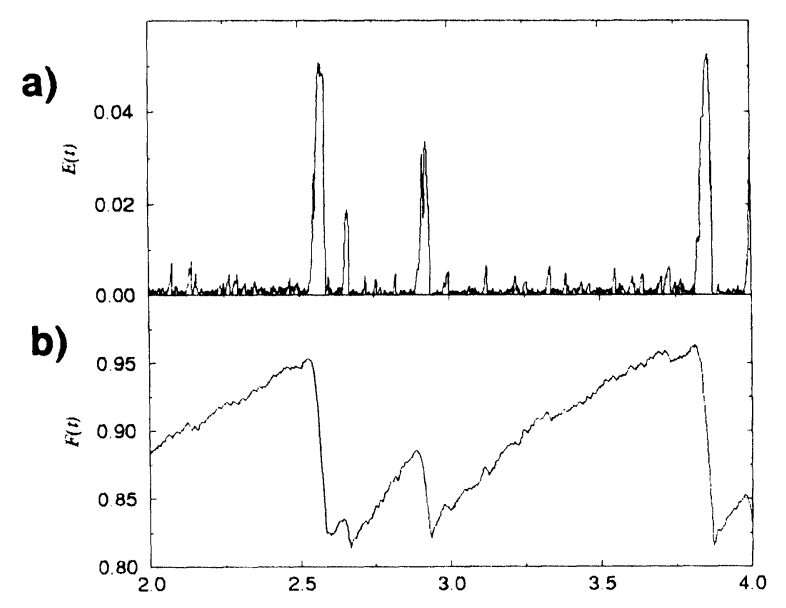

FIGURE 4 Stress relaxation normalized to one cell as a function of time (a); mean value of stress applied to one cell as a function of time (b); plotted for one-dimensional 35block CA with relaxation law (10); $\alpha=3, \delta f=0.01$. 
initial transient period the system is chaotically approaching the statistically steady regime. The dynamical process corresponding to this evolution is characterized by a large number of events with small $E(t)$ and rare events with huge redistribution of stress. The potential function $F(t)$ is presented in Fig. 4(b). As for many other systems with avalanches, it has a saw-like behavior. The closer to the critical state, the higher the probability of the occurrence of strong event.

The comparison of Fig. 4(a) and (b) shows that the events with small $E(t)$ amplitudes (they usually scope only a small number of neighboring cells) just locally redistribute the stress, with increasing total "accumulated stress" $F(t)$ of the automaton. When system is close to the critical state, a small external "push" is sufficient to initiate an avalanche mechanism.

For better understanding let us consider particular example of CA evolution starting at specially prepared initial configurations. In the example (Fig. 5) at $t=0$ all $f_{k}(0)$ except for the central cell were set to $1-\epsilon$, where $\epsilon$ is a small positive parameter. The value of the central cell $f_{k}(0)$ was set larger than for all other cells, but less than 1 .

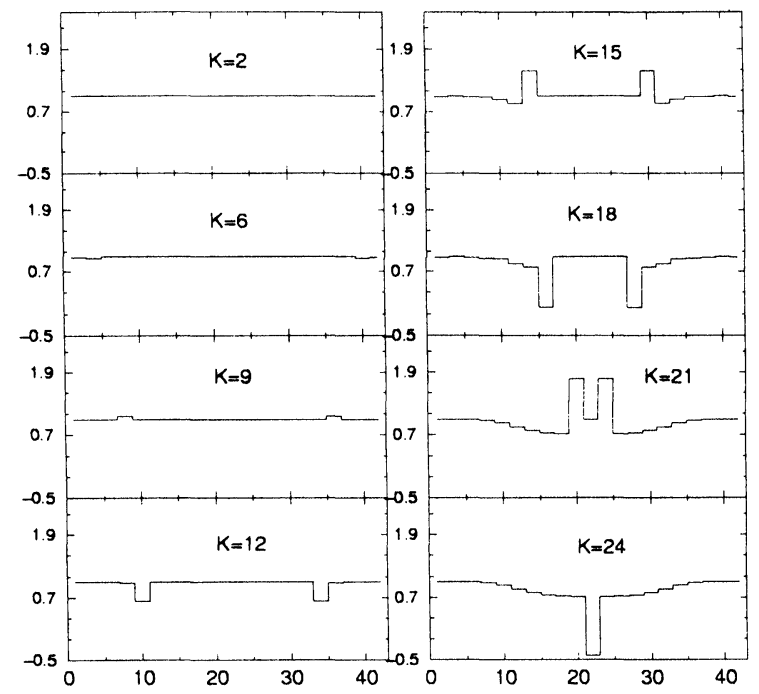

FIGURE 5 The evolution of one-dimensional CA. Plotted for the first initial configuration: 35 -cell $\mathrm{CA}$ with relaxation law (10); $\alpha=3, \delta f=0.01$.
The evolution of this initial configuration is shown in Fig. 5. Two waves originated from the center of the automaton and moving towards its edges are observed. The redistribution of stress in this process increases at each time step and reaches its maximum value at the last step, when two waves collapse in the center of the CA. When the "potential energy" $F(t)$ gains its minimal value, the energy accumulation starts again (due to both the external "pumping", caused by the moving upper plate, and the redistribution of stress inside the system by means of small local events).

In the second example, presented in Fig. 6, the initial states $f_{i}(0)$ of all cells were set to $1-\epsilon$. Figure 6 demonstrates that perturbations arising at the ends of CA produce two waves moving towards each other and which, after collision in the center, suppress each other.

\subsection{Discrete Evolution}

To analyze the dynamical properties of our CA analytically one can present its evolution in a recurrent form $f(t+1)=\psi(f(t), f(t-1), \ldots)$, or

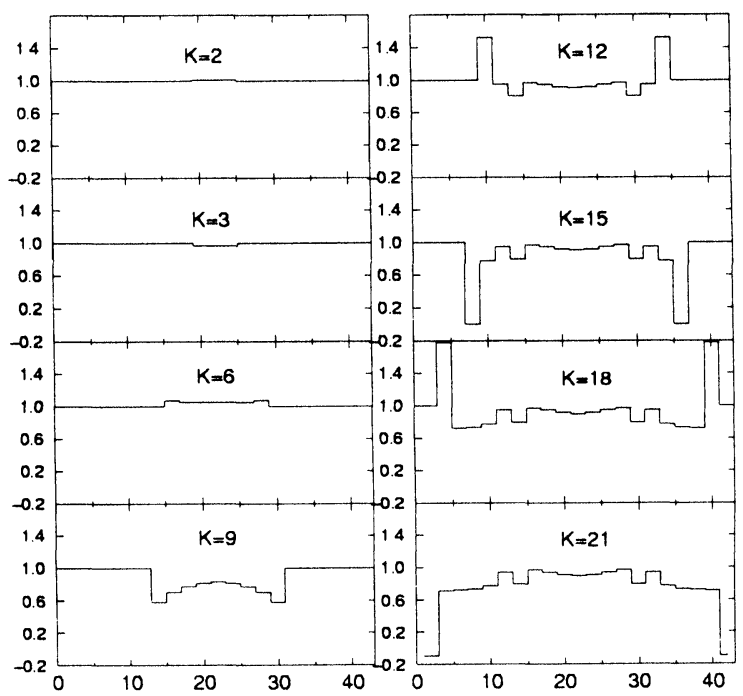

FIGURE 6 The evolution of one-dimensional CA. Plotted for the second initial configuration: 35 -cell $\mathrm{CA}$ with relaxation law $(10) ; \alpha=3, \delta f=0.01$. 
even better in a difference form

$$
f(t+1)=f(t)+\psi(f(t), f(t-1), \ldots),
$$

where $t$ is the discrete time. Fortunately, the twostep process of the CA evolution studied in this article allows representation in a form of a one-step difference equation.

The first step - the seismic event, during which the values of all over-critical cells are redistributed to their neighbors - can be written in matrix notation (with components corresponding to the cells of the automaton):

$$
\vec{F}_{t+1}=\vec{F}_{t}+[A] \vec{G}\left(\vec{F}_{t}\right)
$$

where $\vec{F}_{t}=\left(f_{1}(t), f_{2}(t), \ldots, f_{N}(t)\right)^{\mathrm{T}}$ denotes the state of the automaton at the time of $t$ th iteration. Here we consider one-dimensional CA for simplicity: two-dimensional scheme can be described in the same way. Let

$$
\vec{G}\left(\vec{f}_{t}\right)=\left(g_{1}(t), g_{2}(t), \ldots, g_{N}(t)\right)^{\mathrm{T}}
$$

be the vector of stress release $\left(g_{i}(t) \equiv f_{i}(t)-\right.$ $\left.\phi\left(f_{i}(t)-f_{\text {th }}\right)\right)$, where $\phi$ is the stress relaxation function defined by Eqs. (7) and (9), $[A]$ is $[N \times N]$ matrix

$$
[A]=\left(\begin{array}{ccccccc}
-1 & \alpha & 0 & 0 & 0 & \cdots & 0 \\
\alpha & -1 & \alpha & 0 & 0 & \cdots & 0 \\
0 & \alpha & -1 & \alpha & 0 & \cdots & 0 \\
\cdots & \cdots & \cdots & \cdots & \cdots & \cdots & \cdots \\
0 & \cdots & 0 & \alpha & -1 & \alpha & 0 \\
0 & \cdots & 0 & 0 & \alpha & -1 & \alpha \\
0 & \cdots & 0 & 0 & 0 & \alpha & -1
\end{array}\right)
$$

where $\alpha=k_{\mathrm{c}} /\left(2 k_{\mathrm{c}}+k_{\mathrm{p}}\right)$ according to (6). Since the stress redistribution mechanism (6) is applied at each time step until all cells evolve to underthreshold values, the difference equation (12) can be iterated to express the state of the automaton at the end of the certain event in terms of its state at the beginning of this event:

$$
\vec{F}_{i}^{\mathrm{e}}-\vec{F}_{i}^{\mathrm{b}}=[A] \vec{S}_{i},
$$

where $\vec{S}_{i}=\sum_{t \in i} \vec{G}\left(\vec{F}_{t}\right)$ is the integral stress released in the $i$ th cycle. (Subscript $i$ labels the cycles of the automaton, i.e. the periods of evolution from one pumping (8) to the next.)

At the second step of CA evolution (see also Section 2.3) the values of all cells are uplifted by the tectonic force (8) with the value $\delta=k_{\mathrm{p}} v \Delta t$ :

$$
\vec{F}_{i+1}^{\mathrm{b}}=\vec{F}_{i}^{\mathrm{e}}+\delta \vec{e}_{0},
$$

where $\vec{e}_{0}=(1,1, \ldots, 1)^{\mathrm{T}}$, the superscripts ' $\mathrm{b}$ ' and 'e' denote 'begin' and 'end' of the event, respectively.

Taking into account (14) and the evolution law (13), we can define the "energy functional"

$$
U(\vec{S})=-\frac{1}{2}(\vec{S},[A] \vec{S})-\delta\left(\vec{e}_{0}, \vec{S}\right),
$$

which allows us to rewrite the, evolution equation (13) in the gradient form

$$
\vec{F}_{i+1}^{\mathrm{b}}=\vec{F}_{i}^{\mathrm{b}}-\nabla_{\vec{S}_{i}} U\left(\vec{S}_{i}\right) .
$$

Since $\vec{S}(t)$, which is an argument of the r.h.s. of the latter equation, depends only on the integral stress released up to the time $t$, but does not depend on the particular path of the CA evolution. As we will see below, the "energy functional" may be a more relevant characteristic of events, especially in twodimensional CA.

The functional $U(\vec{S})$ can be considered as an analog of the released energy of the original BK model. In Fig. 7 we present the time dependence of $U(\vec{S}(t))$, calculated for one-dimensional cellular automaton.

\subsection{Asymmetric Redistribution of Stress}

The other point to be stressed here is that one of the basic principles of the construction of cellular automata for physical applications, is the preservation of the symmetry of the original physical system as well as possible. The CA of Bak and Tang [1] and Nakanishi [8] do not meet this requirement. The original $B K$ model is asymmetric with respect 

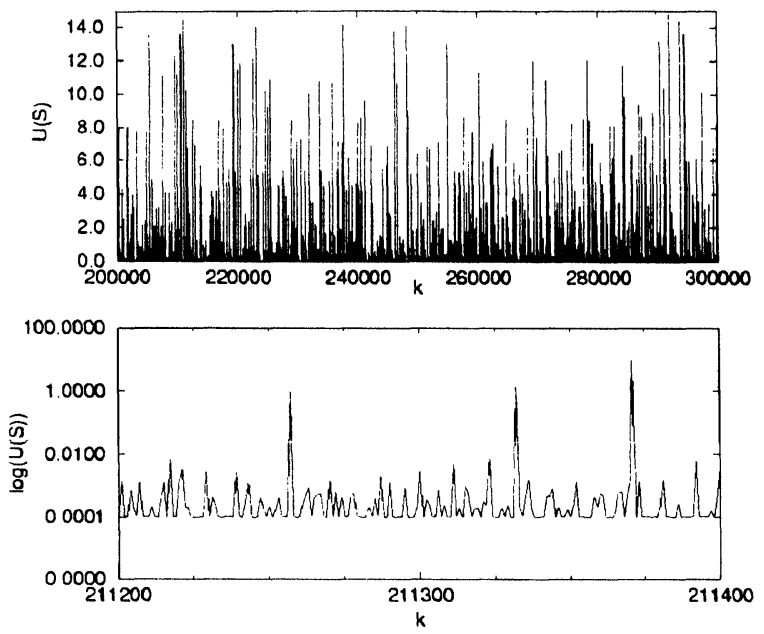

FIGURE 7 The dependence of the energy functional $U(\vec{S})$ on the event number of a one-dimensional 35-cell CA, $\alpha=0.3$.

to the space inversion $x \rightarrow-x$; the driving plate velocity fixes preferable direction. Both CA evidently are not.

The modification of the stress redistribution law which respects the space asymmetry is easily written down:

$$
\begin{aligned}
& f_{i+1} \rightarrow f_{i+1}+(1-\gamma) \frac{2 k_{\mathrm{c}}}{2 k_{\mathrm{c}}+k_{\mathrm{p}}} \delta f_{i}, \\
& f_{i-1} \rightarrow f_{i-1}+\gamma \frac{2 k_{\mathrm{c}}}{2 k_{\mathrm{c}}+k_{\mathrm{p}}} \delta f_{i},
\end{aligned}
$$

where $\gamma=0.5$ corresponds to equal sharing (6), $\gamma=0$ leads to completely asymmetric sharing.

The asymmetric stress redistribution changes the dynamics of the cellular automaton creating preferable direction. The fact of asymmetry has significant physical consequences. Recently, it was even mentioned that an additional asymmetry should be incorporated in BK spring block system itself in order to make it more realistic [9].

We will show now that the asymmetry is an important parameter of the system and affects not only the visually observed dynamics of the avalanches, but also the power-law distribution of events. For this purpose we have performed computer simulations with one-dimensional 35 blocks CA for different values of the asymmetry

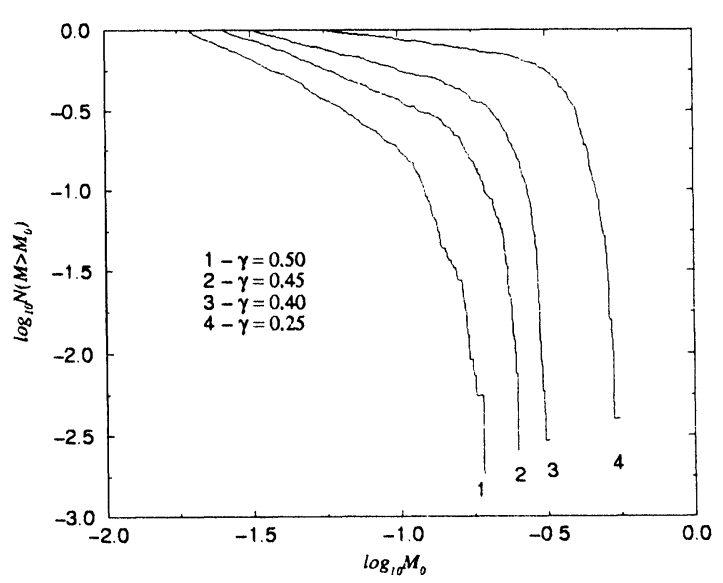

FIGURE 8 Cumulative event distribution $N\left(M>M^{\prime}\right)$ for one-dimensional 35-cell CA. Plotted for different values of asymmetry parameter $\gamma=0.25,0.4,0.45,0.5$. Plotted in logarithmic coordinates vs. the magnitude $m=\log M$ with the event size understood as the total stress relaxation $M=\sum_{i}\left(f_{i}-f_{i}^{\prime}\right)$. The relaxation function $\phi$ was taken as in Nakanishi [8].

parameter $\gamma=0.25,0.4,0.45,0.5$. The cumulative event distribution, i.e. the number of events with the magnitude not less than a given value, which is often expected to have the form of the Gutenberg-Richter law, is presented in Fig. 8.

As it can be seen from the picture, the asymmetry parameter $\gamma$ significantly affects the slope of the curve (the logarithm of the cumulative event number vs. magnitude). We also observe the amplitude of events to decrease with the asymmetry increasing. This means that $\gamma$ is a control parameter of the system, the appropriate choice of which can tune the system close or far from the realistic value of $b \approx 1$ in the Gutenberg-Richter law [3].

\section{BK-LIKE AUTOMATA IN TWO SPACE DIMENSIONS}

In this section we describe the two-dimensional CA, which we construct by modifying the OlamiFeder-Christensen model [10]. The modifications are:

- The stress relaxation function was taken from the one-dimensional Nakanishi CA model [8]. 
- The ratio of compressive and pulling stiffnesses $k_{\mathrm{c}} / k_{\mathrm{p}} \approx 10$ was taken in accordance to corresponding ratio of real earth crust materials.

- The model is made asymmetric in one space direction.

It is shown by numerical simulations that the definition of CA event magnitude, taken by many authors as a sum of stress drop $\sum\left(f-f^{\prime}\right)$, does not give a power-like distribution of events for twodimensional automata, as it does for one-dimensional ones. In our approach, in contrast, an energy functional of second power in stress release gives feasible distributions for both one- and two-dimensions. The asymmetry, which we incorporate in our CA, turns out to be a new control parameter of the system and allows the control of the slope of the event distribution.

Let us present the model in more detail. The specific feature of earthquake faults in comparison to other strong events in continuous media, say, shock waves in gases, is that an earthquake is $a$ relaxation of shear stress, rather than a simple compression as for acoustic waves.

\subsection{The Proposed CA}

A straightforward generalization of the one-dimensional Nakanishi automaton [8] to two space dimensions was given by Olami et al. in [10]. Their system is a two-dimensional array of blocks connected to each other by harmonic springs (with strength $k_{1}$ and $k_{2}$ for two orthogonal directions respectively), see Fig. 9 redrawn from [10]. Each block is attached to the moving upper line by a spring of stiffness $k_{\mathrm{p}}$.

The particular numerical realization of our scheme was the following:

1. Initialize all sites to a random value between 0 and $f_{\mathrm{th}}$.

2. If any $f_{i j} \geq f_{\text {th }}$ then redistribute the force on $f_{i j}$ to its neighbors according to the rules:

$$
\begin{aligned}
f_{i j}^{\prime} & =\phi\left(f_{i j}-f_{\mathrm{th}}\right) \\
f_{i+1, j} & \rightarrow f_{i+1, j}+(1-\gamma) \frac{2 k_{1}}{2 k_{1}+2 k_{2}+k_{\mathrm{p}}} \delta f_{i j},
\end{aligned}
$$

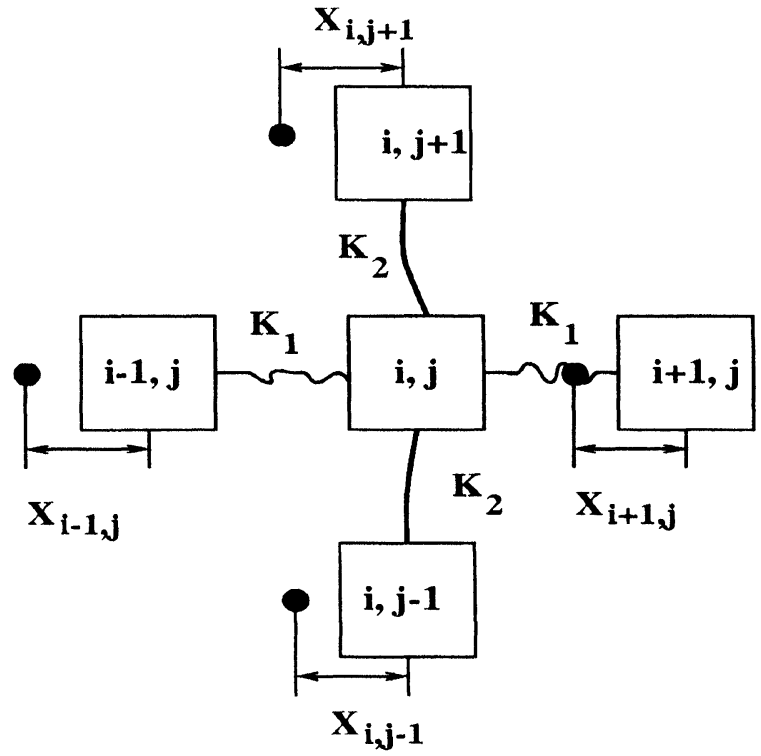

FIGURE 9 Two-dimensional BK-like cellular automaton (redrawn from Ref. [10]).

$$
\begin{aligned}
f_{i-1, j} & \rightarrow f_{i-1, j}+\gamma \frac{2 k_{1}}{2 k_{1}+2 k_{2}+k_{\mathrm{p}}} \delta f_{i j}, \\
f_{i, j \pm 1} & \rightarrow f_{i, j \pm 1}+\frac{k_{2}}{2 k_{1}+2 k_{2}+k_{\mathrm{p}}} \delta f_{i j},
\end{aligned}
$$

where $\delta f_{i j}=f_{i j}-f_{i j}^{\prime}$.

3. Repeat step 2 until the earthquake is fully evolved.

4. Increase the tension of each block according to the rule $f_{i j} \rightarrow f_{i j}+v k_{\mathrm{p}} \Delta t$, where $\Delta t$ is time-step parameter of CA.

Evidently this machine does not account for shear stresses: in terms of Fig. 9 they would correspond to rotations of blocks. However, this automaton has rather intriguing dynamics and we should understand what behavior it describes. To do this, let us recall that the original paper [6] contains a number of physical models, rather than the famous spring-block model alone. The first model (see Figs. 1 and 2 of [6]) is a string which rests on a rough surface moving with constant velocity. The sequence of events is as follows (cited 
from [6, p. 343]):

Starting from some initial configuration, the masses and strings ride along the moving surface without deformation of the chain, except for segments at the ends...

After some critical time, the mass nearest the end is displaced abruptly in the direction opposite to the motion of supporting surface, thereby changing the angles of the segments of the string between two end masses and the support. After this sudden motion cases, the entire system again rides at relative rest on the moving surface...

If we consider a two-dimensional system and suppose the rough material to be elastic and viscous, the velocity of rough material has to depend on the transversal (normal to $\vec{v}$ ) coordinate as well. In a discrete version the situation can be modelled as shown in Fig. 9 of [10], where a system of blocks rests on a rough surface and each block of the system is attached to this surface by harmonic spring of strength $k_{\mathrm{p}}, k_{1}$ corresponds to $k_{\mathrm{c}}$ of the BK model; $k_{2}$ stands for the shear stress, which was introduced in [10] for the first time. The direction of moving surface is the $x$-direction of the picture.

Thus $k_{\mathrm{c}}$ and $k_{\mathrm{p}}$ harmonic springs are exactly that of 1D BK model, while $k_{2}$ stands for the additional shear stress appearing in two dimensions. To have a physically relevant model it is desirable to relate stiffness and shear strength close to the relation of rock materials. This means $k_{1} \approx 3 k_{2}$, and $k_{1} / k_{\mathrm{p}} \gg 1$. The two-dimensional model with symmetric stress redistribution $-k_{1}=k_{2}$ and no asymmetry parameters $(\gamma=0.5)$ included - shows potential behavior, which resembles propagation of shock waves initiated by potential isotropic sources. Such dynamics we can expect to happen for the waves irradiated by point spherically symmetric explosions: by no means such behavior resembles shear waves.

\subsection{Power-Law Distribution of Events}

The direct attempt to obtain the GutenbergRichter power-law distribution of events with a

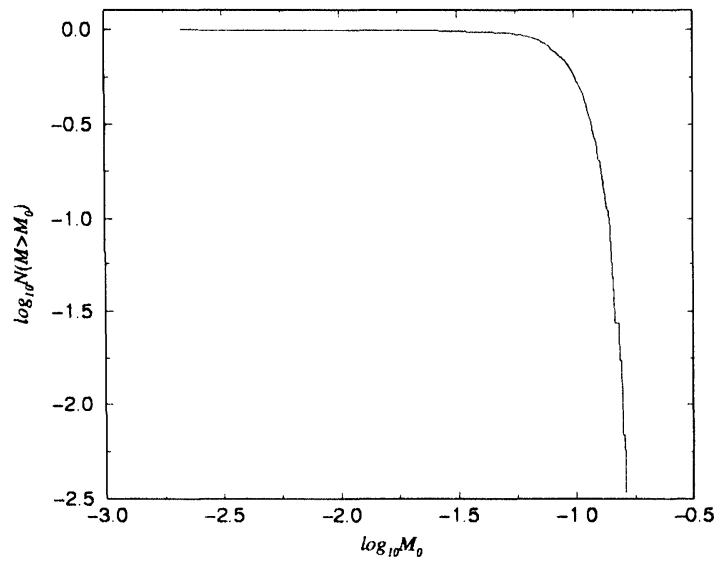

FIGURE 10 The event distribution for our $35 \times 35$-cell CA.

magnitude defined by (10) for our two-dimensional $\mathrm{CA}$, gives a distribution, which is far from linear dependence in logarithmic coordinates, see Fig. 10. The reason for this is that the introduction of $k_{\mathrm{s}}$ "shear" springs makes the relation (10) less relevant than in one dimension. To improve the situation, we redefine the magnitude of event, using the energy functional $U(\vec{S})$. Being quadratic in stress release, see Eq. (15), this functional is less sensitive to the details of stress redistribution, but accounts mainly for the global event characteristics.

The evolution of energy functional $U(\vec{S})$, calculated for two-dimensional cellular automaton is presented in Fig. 11. The stochastic behavior, with a few irregularly distributed huge events (avalanches) is clearly observed there in figures. In the figures below we present the comparison between cumulative event distribution calculated with respect to $U(\vec{S})$ (Fig. 12) and the magnitude (10) (Fig. 10), respectively. Our definition of the magnitude (15), as is observed, provides more clear linear dependence. In comparison to the one-dimensional case, dealing with 2D CA we are restricted to the symmetric $\gamma=0.5$ stress redistribution, but the tension coefficients may be different of course:

$$
\alpha_{1}=\frac{k_{1}}{2 k_{1}+2 k_{2}+k_{\mathrm{p}}}, \quad \alpha_{2}=\frac{k_{2}}{2 k_{1}+2 k_{2}+k_{\mathrm{p}}} .
$$



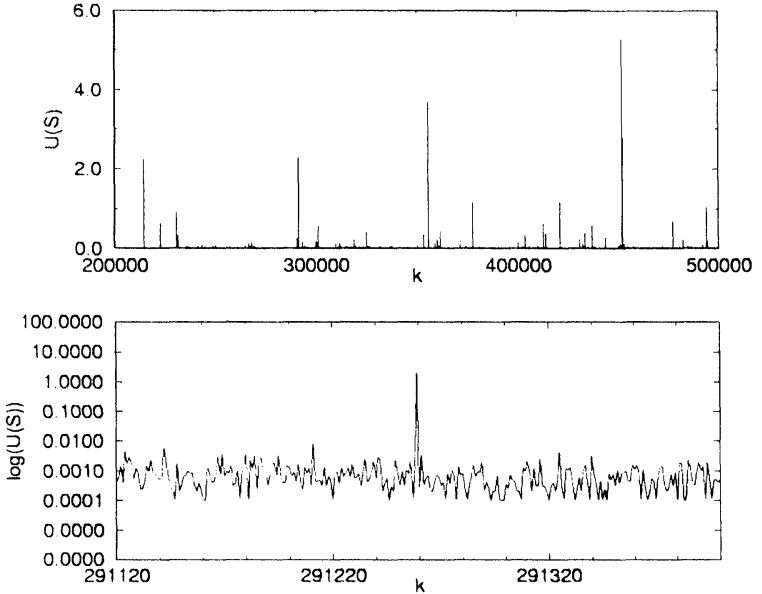

FIGURE 11 Energy functional $U(\vec{S})$ as a function of event number (see explanations in text): two-dimensional $25 \times 15$-cell CA with $\alpha_{1}=0.26, \alpha_{2}=0.087$.

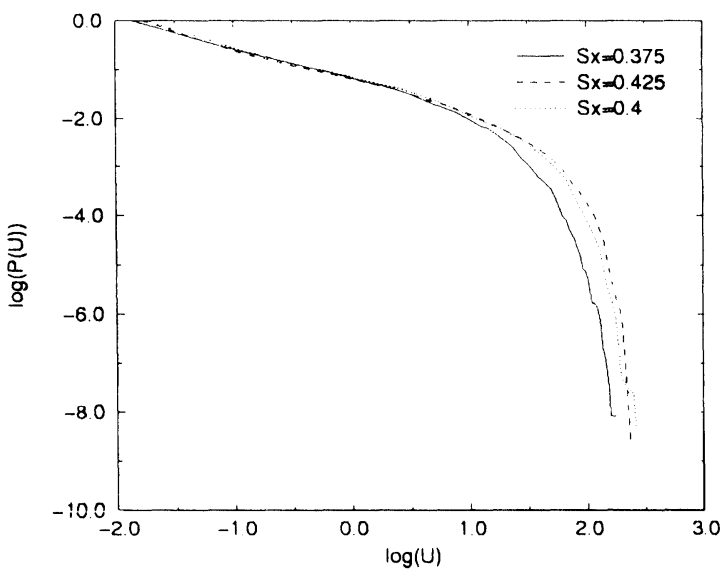

FIGURE 12 The distribution function of event $P(U)$ vs. magnitude $U$; two-dimensional $35 \times 15 \mathrm{CA}$ with different spring tensions. Sx corresponds to $\alpha_{1}, \alpha_{2}$ is chosen in accordance to ratio $k_{1} / k_{2}=3$.

For general case $(\gamma \neq 0.5)$ the evolution laws (18) cannot be expressed in terms of a potential function $U(\vec{S})$, since the existence of the potential $U(\vec{S})$ demands the symmetry of the matrix $A$ (see Eq. (12)) $A_{i j}=A_{j i}$. Physically, this is related to the fact that at least two potentials - scalar $\phi$ and vector $\vec{A}$ - are required to represent an arbitrary vector field in a gradient form $\vec{v}=\nabla \cdot \phi+\nabla \times \vec{A}$, rather than scalar potential alone. The introduction of torsion in our CA model is beyond the scope of this paper [11].

\subsection{Asymmetric Modification of Our CA}

The snapshot of the dynamics for the symmetric automaton $(\gamma=0.5)$ and asymmetric one $(\gamma=0.35)$ is shown in the applet figure below, Figs. 13 and 14. The comparison of this two figures ensures that the asymmetric automaton gives a more realistic picture, since traveling waves are clearly observed

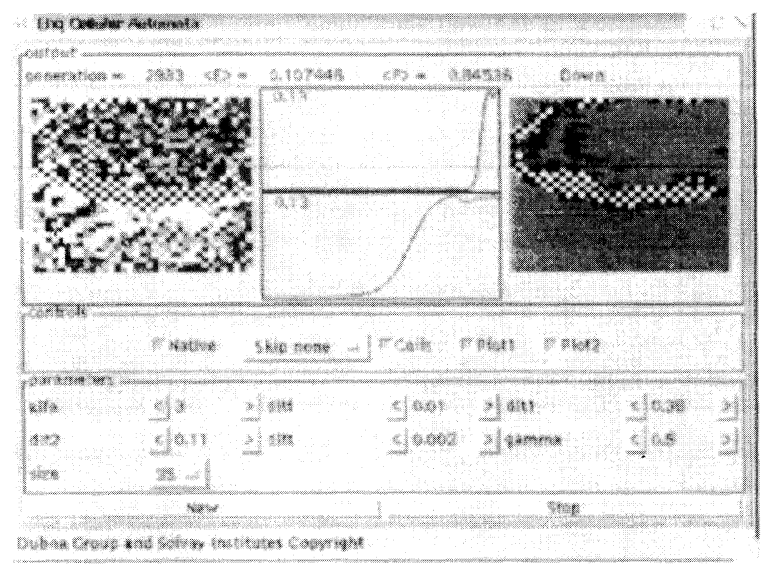

FIGURE 13 Screen-shot of the visualization Java program for two-dimensional CA: symmetric case (available at $/ w w w$ http://inserv.jinr.ru/Projects/ISPRA/ca/index.html $\rangle$ ).

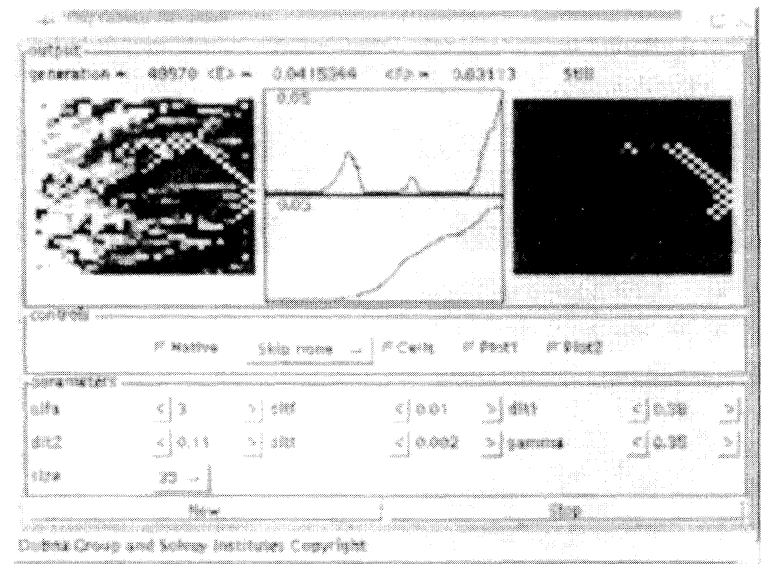

FIGURE 14 Screen-shot of the visualization Java program for two-dimensional CA: asymmetric case, $\gamma=0.35$. 
on it, rather than just bursts of compressions and explosions, as on the first one. The analysis of the cumulative event distributions for one-dimensional CA (see Fig. 8), obtained for different values of $\gamma$ shows that the slope of the distribution (i.e. the $b$ exponent, if the linear Gutenberg-Richter approximation is considered) essentially depends on $\gamma$. So, changing the asymmetry parameter we change the evolution of the cascade observed. This may describe different physical situations.

\section{CONCLUSION}

The ideas of self-organized criticality have suggested the application of CA to the simulation of earthquake related processes. In general, the CA technique is incomparably faster than solving differential equations, but its applicability to an arbitrary system is not granted a priori. For the case of tectonic processes CA seem to be very appropriate. This results from both theoretical and experimental arguments, as well as from the numerical simulations. In both CA simulations and real earthquake processes the power-law distribution of events $N\left(E>E_{0}\right)=E^{-B}$, with $1<B<2$ is clearly observed. Whether or not earthquakes are SOC phenomena in mathematical sense (the $N \rightarrow \infty$ limit may not be appropriate in geophysics), the tree-like cascade structures evidently take place in both. Besides this qualitative similarity, as we observed in our investigation, the value of the Hurst exponent calculated over CA generated time series (see Fig. 15) is about $h=0.6-0.7$, which is close to the values obtained in field observations [12].

We attempted to construct a cellular automaton, which is as close to the existing dynamical models (usually written in the language of differential equations) as possible. We proposed an asymmetric stress redistribution CA which better fits the symmetry of the original BK model. We have also developed an analytic method which suggests a new integral characteristic of the CAgenerated event size.

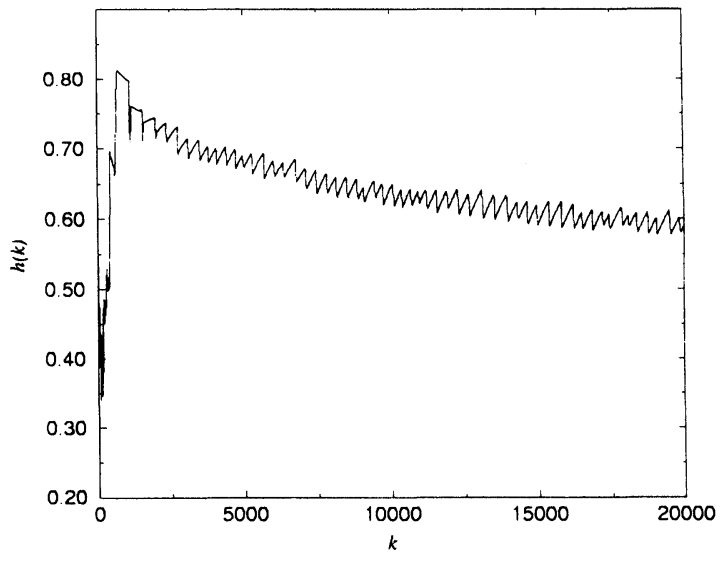

FIGURE 15 The Hurst exponent for the time series generated by two-dimensional CA.

The next step towards the construction of the more realistic CA for the earthquake-related studies should account for the fact that seismic events are essentially shear events, and, therefore, new degrees of freedom should be incorporated in the numerical models. An analytical investigation of the problem might require some new links between dynamical models, SOC theory and phase transitions [13].

\section{Acknowledgments}

The authors are grateful to Profs. G. Molchan, M. Shnirman and V. Priezzhev for useful discussions and to Prof. I. Prigogine for his enthusiastic support.

This work was supported by the European Commission through the collaboration of the International Solvay Institutes with the Joint Research Center, Ispra and by the Belgian Government through the Interuniversity Attraction Poles.

\section{References}

[1] P. Bak and C. Tang. Earthquake as a self-organized critical phenomenon. Journal of Geophysical Research, 94(B11): 15635-15637, 1989.

[2] P. Bak, C. Tang and K. Wiesenfeld. Self-organized criticality. Phys. Rev. A, 38(11): 364-374, 1989. 
[3] B. Gutenberg and C.F. Richter. Frequency of earthquakes in California. Bulletin of the Seismological Society of America, 34: 185-188, 1944.

[4] B. Mandelbrot. Fractals: Form, Chance and Dimension. Freeman and Co., San Francisco, 1977.

[5] V.V. Zosimov and L.M. Lamshev. Fractals in wave processes. Uspekhi Fiz. Nauk, 165(4): 361-402, 1995 (in Russian).

[6] R. Burridge and L. Knopoff. Model and theoretical seismicity. Bulletin of the Seismological Society of America, 57(3): 341-371, 1967.

[7] J.M. Carlson and J.S. Langer. Mechanical model of an earthquake fault. Phys. Rev. A, 40(11): 6470-6484, 1989.

[8] H. Nakanishi. Statistical properties of the cellular-automaton model for earthquakes. Phys. Rev. A, 43(12): 6613$6621,1991$.
[9] J. Schmittbuhl, J.P. Vilotte and R. Roux. Velocity weakening friction: A renormalization approach. Journal of Geophysical Research, 101(B6): 13911-13917, 1996.

[10] Z. Olami, H.S. Feder and K. Christensen. Self-organized criticality in a continuous, nonconservative cellular automaton modelling earthquakes. Phys. Rev. Lett., 68(8): 1244-1246, 1992.

[11] W. Tworzydlo and O. Hamzeh. On the importance of normal vibrations in modelling of stick slip in rock sliding. Journal of Geophysical Research, 102(B7): 15091-15103, 1997.

[12] C. Lomnitz. Fundamentals of Earthquake Predictions. John Wiley and Sons Inc., Ashurst, UK, 1994.

[13] L. Gil and D. Sornette. Landau-Ginzburg theory of selforganized criticality. Phys. Rev. Lett., 76(21): 3991-3994, 1996. 


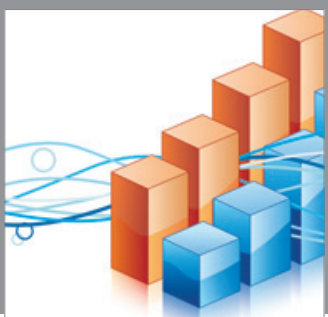

Advances in

Operations Research

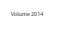

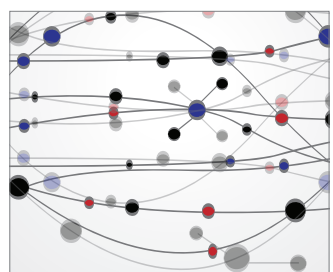

\section{The Scientific} World Journal
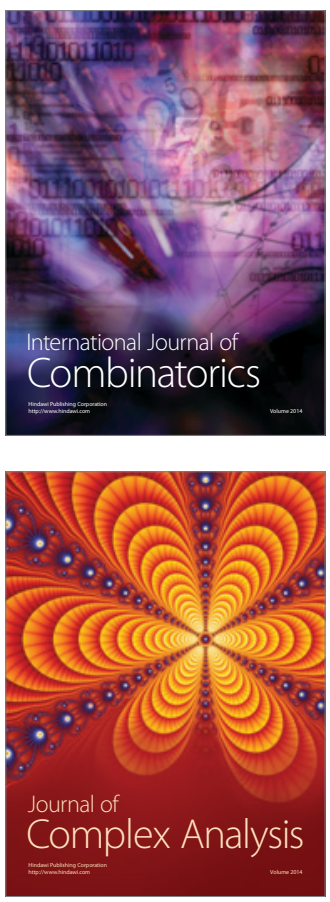

International Journal of

Mathematics and

Mathematical

Sciences
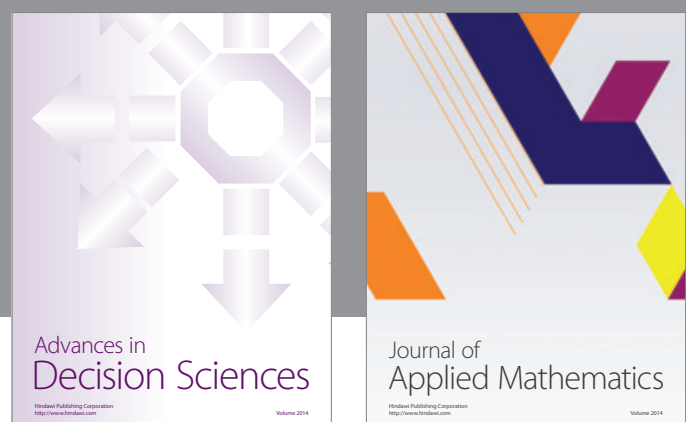

Journal of

Applied Mathematics
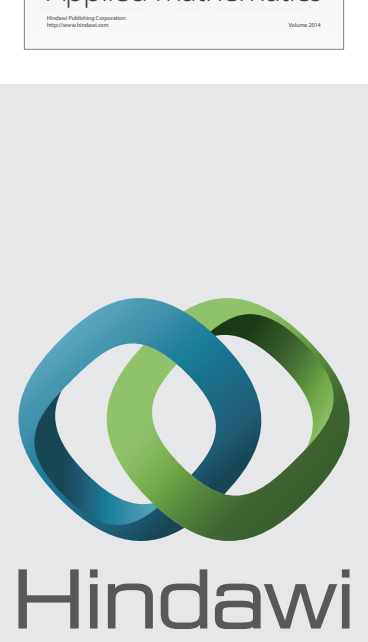

Submit your manuscripts at http://www.hindawi.com
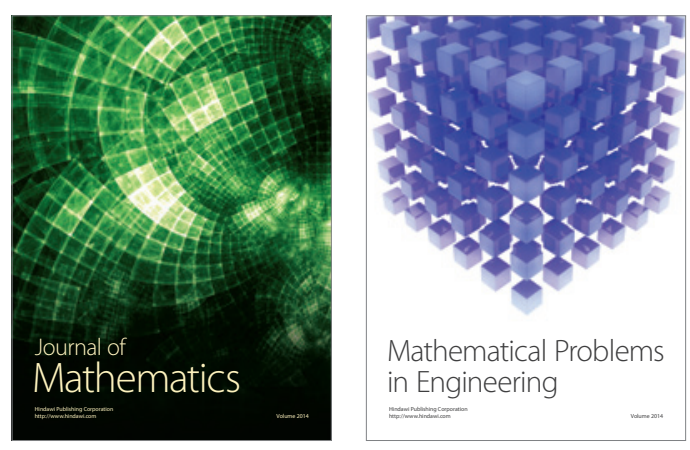

Mathematical Problems in Engineering
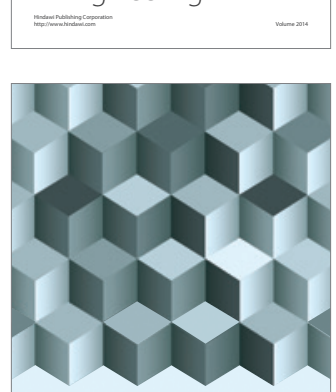

Journal of

Function Spaces
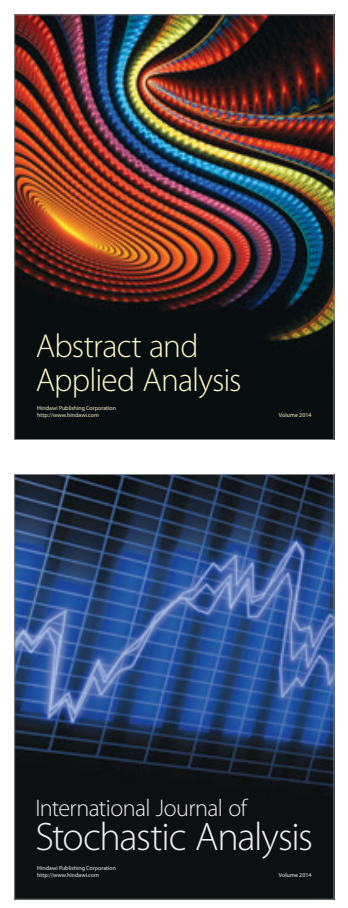

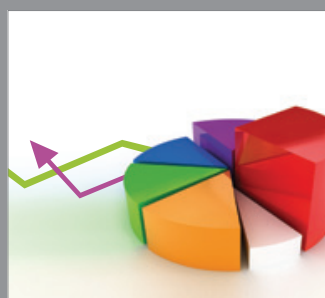

ournal of

Probability and Statistics

Promensencen
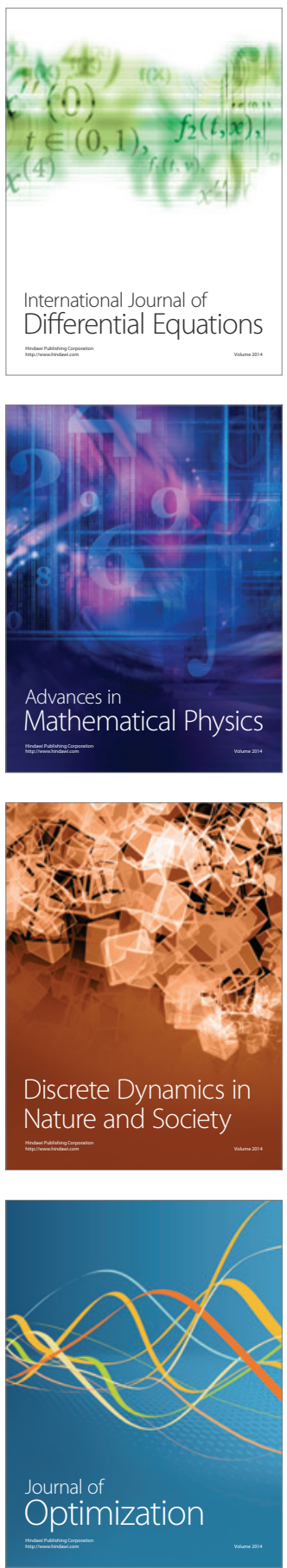